\title{
Fatores socioeconômicos associados à expansão de casos da COVID-19 no Rio Grande do Sul
}

\author{
Diego Pierotti Procópio*
}

Departamento de Zootecnia e Extensão Rural, Universidade Federal de Mato Grosso, Cuiabá, MT, Brasil

Histórico do Artigo:
Recebido em
12/08/2020
Aceito em
$25 / 09 / 2020$

Palavras-chave: Auxílio emergencial; COVID-19; pobreza; PNAE; segurança alimentar e nutricional

\begin{abstract}
RESUMO
Objetivou-se verificar quais indicadores socioeconômicos possuem a capacidade de diferenciar os grupos de municípios sul-rio-grandenses entre os níveis de ocorrências de casos acumulados de COVID-19 acima da média estadual (superior a 628,30/100 mil hab) e abaixo da média. A metodologia utilizada foi a Análise Discriminante e as variáveis selecionadas foram a taxa de casos acumulados da COVID-19, taxa de pobreza, nível de informalidade, densidade de pessoas por dormitório no domicílio e o acesso aos serviços de abastecimento de água e coleta de esgoto. As bases de dados utilizadas foram o painel coronavírus RS da Secretária de Saúde do Estado do Rio Grande do Sul e o Censo Demográfico de 2010 do Instituto Brasileiro de Geografia e Estatística (IBGE). Dentre os principais resultados alcançados, verificou-se que a expansão de casos da COVID-19 nos municípios do Rio Grande do Sul está relacionada à renda, representada pelas variáveis de taxa de pobreza e nível de informalidade. Sendo assim, destacam-se a importância de políticas públicas de transferência de renda direta, como é o caso do auxílio emergencial, e de garantia da segurança alimentar e nutricional por meio de acesso a uma alimentação saudável para minimizar a expansão dos casos de COVID-19 nos municípios sul-riograndenses.
\end{abstract}

Socioeconomic factors associated of expansion of COVID-19 cases in Rio Grande do Sul

\section{ABSTRACT}

The study aims to verify which socioeconomic indicators have the capacity to differentiate the groups of municipalities in Rio Grande do Sul between the levels of occurrences of accumulated cases of COVID-19 above the state average (above 628.30/100 thousand inhabitants) and below average. The methodology used was the Discriminant Analysis and the selected variables were the tax of accumulated cases of COVID-19, poverty tax, level of informality, density of people per dormitory at domicile and access to water supply and sewage collection services. The databases used were the Coronavirus RS panel of the Secretary of Health of the State of Rio Grande do Sul and the 2010 Demographic Census of the Brazilian Institute of Geography and Statistics (IBGE). Among the main results achieved, it was found that the expansion of COVID-19 cases in the municipalities of Rio Grande do Sul is related to income, represented by the variables of poverty rate and level of informality. Therefore, the importance of public policies for direct income transfer, such as emergency aid, and the guarantee of food and nutrition security through access to healthy food to minimize the expansion of COVID- 19 in the municipalities of Rio Grande do Sul.

\section{Introdução}

O CoronaVirus 2 (SARS-Cov2) é o patógeno da COVID-19 e foi inicialmente diagnosticada em dezembro de 2019 na cidade de Wuhan, na China. A partir da expansão da doença nos países através do aumento do número de casos e óbitos, a Organização Mundial de Saúde (OMS) declarou que o evento constituía uma Emergência de Saúde Pública de Importância Internacional (ESPII) no dia 30 de janeiro de 2020. No dia 11 de março de 2020, com a notificação de mais de 110 mil casos e 4 mil óbitos em diferentes países de todos os continentes, a OMS declarou a pandemia de

\footnotetext{
* Autor correspondente: diego_pierottivrb@yahoo.com.br (Procópio D.P.)
} 
COVID-19 (1). No dia 05 de agosto de 2020, o número de casos confirmados no mundo atingiu um patamar de 18,61 milhões de pessoas e 702,64 mil óbitos em 215 países (2).

O primeiro caso de contaminação por COVID-19 no Brasil foi em 26 de fevereiro de 2020 (3). No dia 06 de agosto foram contabilizados um total de 2,91 milhões de casos e 98,49 mil óbitos no país. Dentre as regiões brasileiras, o maior número de casos acumulados foi no Sudeste (1,00 milhão), seguido do Nordeste (922,46 mil), Norte (434,06 mil), Centro-Oeste (286,83 mil) e Sul (264,74 mil). As unidades federativas que apresentaram o maior número de casos acumulados foram São Paulo (598,67 mil), Ceará (183,30 mil), Bahia (183,69 mil), Rio de Janeiro (174,06 mil) e Minas Gerais (142,82 mil) (4).

O Brasil apresenta uma heterogeneidade espacial em termos de demografia, distribuição etária, acessos aos serviços públicos (saneamento básico, saúde, educação etc.) e indicadores de pobreza. Em virtude dessas desigualdades regionais, a epidemia do COVID-19 deve impactar a população brasileira de maneira diferente e em períodos distintos, em virtude de fatores como a transmissibilidade, letalidade e vulnerabilidade socioeconômica da população (5).

Em 2010, os estados de Santa Catarina, Rio Grande do Sul e Paraná tiveram uma taxa de pobreza $^{1}$ de $3,65 \%, 6,37 \%$ e $6,46 \%$, respectivamente. Por sua vez, os estados das regiões Norte e Nordeste possuíam uma parcela maior da população em condição de restrição de renda. As maiores taxas foram representadas por Maranhão (39,53\%), Alagoas (34,29\%), Piauí (34,11\%), Pará (32,33\%), Amazonas (30,78\%), Ceará $(30,32 \%)$ e Acre $(29,46 \%)(6)$.

Existe também uma desigualdade entre as unidades federativas brasileiras no acesso ao serviço de esgotamento sanitário (2). No ano de 2010, as maiores taxas foram de São Paulo (85,93\%), Distrito Federal (78,91\%), Minas Gerais (74,63\%), Espírito Santo $(66,66 \%)$ e Paraná $(52,18 \%)$, representando as regiões Centro-Oeste, Sul e Sudeste. Já as menores taxas foram de Rondônia (5,85\%), Amapá (6,12\%), Piauí (7,24\%), Pará $(9,47 \%)$, Maranhão $(10,77 \%)$ e Tocantins $(12,89 \%)$ que estão localizados nas regiões Nordeste e Norte (6).

O insuficiente conhecimento científico sobre o vírus da COVID-19, a alta taxa de disseminação e a capacidade de provocar mortes em populações que vivem em condições de vulnerabilidade socioeconômica, geram incertezas sobre os tipos de estratégias que devem ser utilizadas para o enfrentamento da pandemia em diversas partes do mundo. No Brasil, os desafios ainda são maiores em virtude do elevado nível de desigualdade social, onde uma parte considerável da população vive em condições precárias de habitação e com dificuldades de acesso à renda (7).

Dessa forma, para que haja uma melhor compreensão da transmissão do vírus da COVID-19 em um território, é necessário levar em consideração a distribuição e as características socioeconômicas da população em determinada localidade. Em algumas unidades federativas brasileiras, observa-se uma expansão na quantidade de novos casos e óbitos pelo CoronaVirus 2 a partir de julho de 2020, como é o caso das regiões Sul e Centro-Oeste do Brasil (4).

No estado do Rio Grande do Sul, o primeiro caso foi registrado no dia 29 de fevereiro de 2020 e se expandiu para 12.538 casos no dia 01 de junho. Já no dia 06 de agosto foram registrados um total de 78.837 casos, o que representou um aumento de 528,78\% em comparação ao início de junho. Além disso, o total de óbitos registrados foi de 2.231 até o dia 06 de agosto (8).

Em 471 municípios do Rio Grande do Sul havia pelo menos um caso registrado de

${ }^{1}$ Percentual da população com renda domiciliar per capita mensal igual ou inferior a R\$140,00; em valores de agosto de 2010 (6). 
COVID-19 até o dia 06 de agosto de 2020, o que representa em 94,76\% do total de cidades sul-rio-grandenses. Sendo assim, os municípios que tinham o maior número de casos acumulados foram Porto Alegre com 9.535 (incidência de 642,40/100 mil habitantes - hab), Passo Fundo com 4.032 (1.938,50/100 mil hab), Caxias do Sul com 3.387 (662,90/100 mil hab), Novo Hamburgo com 2.821 (1.143,30/mil hab) e São Leopoldo com 2.561 (1.119,30/100 mil hab). A incidência de casos acumulados do estado foi de 692,90/100 mil habitantes no mesmo período (8).

Os municípios sul-rio-grandenses são heterogêneos em termos de indicadores socioeconômicos, como o acesso da população aos serviços públicos (saneamento básico, educação, saúde etc.) (9) e nos índices de pobreza (10). A determinação de quais fatores socioeconômicos estão contribuindo para a expansão dos casos de COVID-19 nos municípios do Rio Grande do Sul se torna relevante no que tange ao desenvolvimento de políticas públicas de enfrentamento à pandemia provocada pelo vírus SARS-CoV-2.

Diante de tais considerações, objetivou-se verificar quais os indicadores socioeconômicos que mais discriminam os grupos de munícipios sul-rio-grandenses entre os níveis de ocorrências de casos acumulados de COVID-19 acima da média estadual (superior a 628,30/100 mil hab) e abaixo da média (inferior a 628,3/100 mil hab).

\section{Métodos}

A análise discriminante é uma técnica estatística que auxilia na identificação de variáveis que possuem a capacidade de diferenciar grupos previamente definidos e investiga qual variável possui o maior poder de discriminação entre os grupos. A equação ou função linear discriminante assemelha-se a uma equação de regressão múltipla, sendo composta de variáveis independentes que representam as características do elemento, que são ponderadas pelo nível de sua importância ou impacto que causam no resultado (ou variável dependente). A Função Discriminante pode ser representada a partir da Equação 1 (11).

$$
Y=a+b_{1} X_{1}+b_{2} X_{2}+\cdots+b_{n} X_{n}
$$

Em que:

$Y$ é a variável dependente categórica, que indica a pontuação ou o escore discriminante;

a é o intercepto da função quando todo $X_{i}=0$;

$b_{n}$ é o coeficiente discriminante ou a capacidade de cada variável independente possui de discriminar os grupos; e,

$X_{n}$ são os valores das variáveis independentes.

A variável $\mathrm{Y}$ assumi o valor 1 para o grupo de municípios sul-rio-grandenses que possuíam uma taxa de incidência de casos acumulados de COVID-19 acima da média estadual $(628,3 / 100 \mathrm{mil}$ hab) e o valor 0 foi direcionado para o grupo de municípios que possuíam uma taxa de casos acumulados abaixo da média estadual. A base de dados utilizada para a obtenção das informações dos casos de COVID-19 nos municípios sulrio-grandenses foi da Secretária de Saúde do Estado do Rio Grande do Sul (8). Já as variáveis $X_{n}$ são representadas pelos indicadores socioeconômicos do Censo Demográfico de 2010 elaborado pelo Instituto Brasileiro de Geografia e Estatística (Quadro 1). 
Quadro 1 - Indicadores socioeconômicos selecionados para a Análise Discriminante

\begin{tabular}{|l|l|l|}
\hline \multicolumn{1}{|c|}{ Variável } & Sigla & \multicolumn{1}{c|}{ Descrição } \\
\hline Pobreza (12) & POB & $\begin{array}{l}\text { Proporção de indivíduos com renda domiciliar per capita } \\
\text { mensal igual ou inferior a R } \$ 140,00 \text {, em valores de agosto } \\
\text { de 2010. }\end{array}$ \\
\hline $\begin{array}{l}\text { Informalidade } \\
\text { (12) }\end{array}$ & INF & $\begin{array}{l}\text { Razão entre o total de pessoas de 18 anos ou mais sem } \\
\text { ensino fundamental completo e em ocupação informal e a } \\
\text { população total nesta faixa etária, multiplicada por 100. A } \\
\text { ocupação informal é representada por trabalhadores que } \\
\text { não possuam carteira assinada e que não sejam militares, } \\
\text { empregados pelo regime jurídico dos funcionários públicos, } \\
\text { empregadores e trabalhadores por conta própria com } \\
\text { contribuição ao instituto de previdência social. }\end{array}$ \\
\hline $\begin{array}{l}\text { Densidade de } \\
\text { pessoas } \\
\text { dormitório (12) }\end{array}$ & DEN & $\begin{array}{l}\text { Razão entre a população que vive em domicílios com } \\
\text { densidade superior a 2 a população total, multiplicada por } \\
100 . \text { A densidade do domicílio é dada pela razão o total de } \\
\text { moradores do domicílio e o número total de cômodos que } \\
\text { são utilizados como dormitório. }\end{array}$ \\
\hline $\begin{array}{l}\text { Água } \\
\text { canalizada (13) }\end{array}$ & AGC & $\begin{array}{l}\text { Percentual de pessoas que vivem em domić́lio que possui } \\
\text { acesso à água canalizada em pelo menos um cômodo. }\end{array}$ \\
\hline $\begin{array}{l}\text { Esgotamento } \\
\text { sanitário (13) }\end{array}$ & ESS & $\begin{array}{l}\text { Percentual de pessoas que vivem em domićlio que tem } \\
\text { esgotamento sanitário do tipo rede geral de esgoto ou } \\
\text { pluvial. }\end{array}$ \\
\hline
\end{tabular}

As informações dos casos acumulados de COVID-19 dos municípios são do dia 03 de agosto de 2020 e o município de Pinto Bandeira não foi incluído na Análise Discriminante pela ausência de dados socioeconômicos na base de dados do IBGE. O município foi criado no dia 01 de janeiro de 2013 (14), período após o desenvolvimento do Censo Demográfico de 2010. Sendo assim, foram avaliados o total de 496 municípios gaúchos para o alcance do objetivo proposto.

A análise discriminante possui como pressupostos básicos a normalidade multivariada, linearidade, ausência de outliers, ausência de multicolineariedade e a homogeneidade das matrizes de variância co-variância. Para a estimação e teste da qualidade da função discriminante, foram realizados os testes de igualdade das médias das variáveis entre os grupos que procura identificar quais variáveis são as melhores discriminadoras dos grupos analisados. Além disso, o segundo teste denominado de Qui-Quadrado, mostra se realmente a função serve para discriminar os grupos previamente estabelecidos (11). O nível de significância utilizado para a realização dos testes foi de $1 \%$ ( $p$-valor de $0,01)$.

Por fim, foi realizado o teste que verifica o percentual de classificação correta do modelo (grau de previsão do modelo). A Equação 2 busca determinar o percentual mínimo de classificação correta dos grupos distintos a partir da quantidade de observações (11).

$$
C_{p r o}=p^{2}+(1-p)^{2}
$$

Em que:

$C_{\text {pro }}$ critério de chances proporcionais; 
$p$ proporção de indivíduos do primeiro grupo (em porcentagem em relação ao total); e, $1-p$ proporção de indivíduos do segundo grupo (em porcentagem em relação ao total).

A classificação correta do modelo deve assumir um valor superior ao $C_{\text {pro }}$ dos grupos avaliados para que o resultado seja satisfatório (11).

\section{Resultados}

Na Figura 1 são apresentadas informações sobre a distribuição de municípios sul-riograndenses entre os grupos 0 (abaixo da média estadual) e 1 (acima da média estadual) para a taxa de incidência de casos acumulados da COVID-19 até o dia 03 de agosto de 2020.

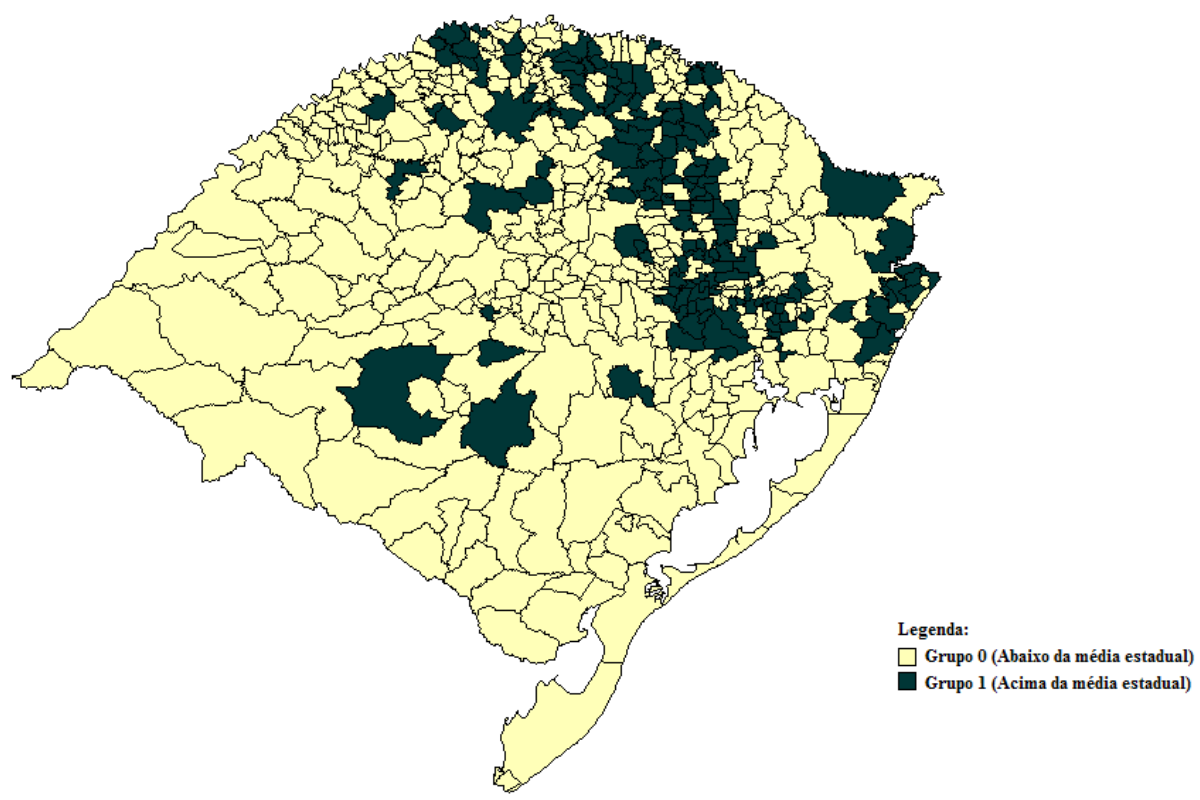

Figura 1 - Distribuição de municípios sul-rio-grandenses entre os grupos 0 e 1 .

Do total de 496 municípios gaúchos, cerca de 69,35\% (corresponde a 344) foram inseridos no grupo 0 e 30,65\% (cerca de 152) foram incluídos no grupo 1 . Dessa forma, a maior parte das localidades analisadas possuíam uma taxa de incidência de casos acumulados da COVID-19 inferior à média estadual.

$\mathrm{Na}$ Tabela 1 são apresentadas as estatísticas descritivas dos indicadores socioeconômicos nos municípios sul-rio-grandenses que compõem os grupos 0 e 1 .

Tabela 1 - Estatística descritiva dos indicadores socioeconômicos dos 496 municípios sul-rio-grandenses

\begin{tabular}{|c|c|c|c|}
\hline Indicador & Mínimo & Máximo & Média \\
\hline $\mathrm{POB}(\%)^{12}$ & 0,19 & 43,55 & 8,91 \\
\hline $\operatorname{INF}(\%)^{12}$ & 17,34 & 76,96 & 43,18 \\
\hline $\operatorname{DEN}(\%)^{12}$ & 0,65 & 50,43 & 12,27 \\
\hline $\operatorname{AGC}(\%)^{13}$ & 60,59 & 100,00 & 97,32 \\
\hline $\operatorname{ESS}(\%)^{13}$ & 0,00 & 85,85 & 15,39 \\
\hline
\end{tabular}

Nota: POB - taxa de pobreza; INF - parte da população com idade superior a 18 anos sem ensino fundamental completo e com ocupação na informalidade; DEN - densidade de pessoas por dormitório no domicílio; AGC - acesso ao serviço de água encanada; ESS - acesso ao serviço de esgotamento sanitário. 
O serviço de abastecimento de água canalizada (97,32\%) apresentou uma média superior ao esgotamento sanitário $(15,39 \%)$ nos municípios analisados. A taxa de pobreza e a densidade por dormitório superior a 2 foram as que apresentaram as menores médias dentre os indicadores socioeconômicos selecionados, sendo de 8,91\% e $12,27 \%$, respectivamente. As informações da população com idade de 18 anos ou mais sem ensino fundamental completo e com ocupação na informalidade entre os municípios sul-rio-grandenses apresentaram um valor médio de 43,18\% e um valor máximo de 76,96\% (Tabela 1). Ou seja, em alguns municípios analisados, a maior parte da população (com idade superior a 18 anos) possui um baixo nível de escolaridade e a ocupação no mercado informal.

Os indicadores socioeconômicos que mais diferenciaram os grupos de municípios sulrio-grandenses de acordo com a taxa de casos acumulados da COVID-19 estão relacionados à taxa de pobreza da localidade e pela população com idade superior a 18 anos com baixo nível de escolaridade e com ocupação na informalidade, que são representadas pelas variáveis POB e INF (Tabela 2).

Tabela 2 - Indicadores socioeconômicos que contribuíram para a diferenciação dos grupos de municípios sul-rio-grandenses com taxas de ocorrência da COVID-19 acima e abaixo da média estadual

\begin{tabular}{cccc}
\hline Indicador & Lambda de Wilks & Estatística $\mathbf{F}$ & $\begin{array}{c}\text { Nível de } \\
\text { Significância } \\
(\boldsymbol{p} \text {-valor })\end{array}$ \\
\hline POB & 0,963 & 18,836 & $\mathbf{0 , 0 0 0} *$ \\
INF & 0,952 & 24,923 & $\mathbf{0 , 0 0 0 *}$ \\
DEN & 0,993 & 3,515 & 0,061 \\
AGC & 0,999 & 0,503 & 0,478 \\
ESS & 0,999 & 0,715 & 0,398 \\
\hline
\end{tabular}

Nota: POB - taxa de pobreza; INF - parte da população com idade superior a 18 anos sem ensino fundamental completo e com ocupação na informalidade; DEN - densidade de pessoas por dormitório no domicílio; AGC - acesso ao serviço de água encanada; ESS - acesso ao serviço de esgotamento sanitário. * Nível de significância de $1 \%$ (p-valor de 0,01 ).

Estatística $\mathrm{F}$ - busca verificar o comportamento das médias das variáveis entre os grupos avaliados. A hipótese nula é de que as médias das variáveis sejam iguais. Dessa forma, é ideal que na Análise Discriminante as médias das variáveis nos grupos sejam diferentes (15).

As unidades de medida do Lambda de Wilks e da Estatística F são em valores numéricos (15).

Após a indicação das variáveis que possuem a capacidade de diferenciar os grupos previamente estabelecidos, foi necessário verificar se a função é apropriada para a Análise Discriminante por meio do teste do Qui-quadrado (Tabela 3).

Tabela 3 - Teste de significância da função discriminante

\begin{tabular}{ccccc}
\hline Teste de funções & Lambda de Wilks & $\begin{array}{c}\text { Qui- } \\
\text { quadrado }\end{array}$ & $\begin{array}{c}\text { Graus de } \\
\text { liberdade }\end{array}$ & $\begin{array}{c}\text { Nível de } \\
\text { Significância } \\
(\boldsymbol{p} \text {-valor })\end{array}$ \\
\hline 1 & 0,939 & 30,718 & 5 & $0,000^{*}$ \\
\hline
\end{tabular}

Nota: * Nível de significância de $1 \%$ (p-valor de 0,01$)$.

As unidades de medida do Lambda de Wilks, Qui-quadrado e dos Graus de liberdade são em valores numéricos (15).

Pelo teste do Qui-quadrado, a função é significativa ao nível de $1 \%$ de significância ( $p$-valor de 0,01), sendo adequada para a Análise Discriminante (11). A função discriminante padronizada é apresentada na Tabela 4. 
Tabela 4 - Função discriminante padronizada para as variáveis selecionadas

\begin{tabular}{cc}
\hline Variáveis & Coeficiente \\
\hline POB & 0,390 \\
INF & 0,830 \\
\hline
\end{tabular}

Nota: POB - taxa de pobreza; INF - parte da população com idade superior a 18 anos sem ensino fundamental completo e com ocupação na informalidade.

A unidade de medida do coeficiente da função discriminante é em valor numérico.

A função discriminante padronizada é composta pelas variáveis PRO e INF que possuem a capacidade de diferenciar os grupos de municípios sul-rio-grandenses com taxas de ocorrências de casos da COVID-19 acima e abaixo da média do estado (Tabela 4). Os coeficientes das variáveis são definidos como pesos discriminantes e atribuem a importância relativa de cada indicador na função discriminante (15). Quanto ao grau de previsão do modelo, o percentual de classificação correta dos grupos foi de $70,4 \%$ e é considerado satisfatório, já que o $C_{\text {pro }}$ dos grupos analisados foi de $57,22 \%$.

\section{Discussão}

A partir da função discriminante padronizada foi possível verificar que a taxa de pobreza e a parcela da população com idade superior a 18 anos sem ensino fundamental completo e com ocupação no mercado informal apresentaram capacidade significativa de diferenciar os grupos de municípios sul-rio-grandenses com taxas de ocorrência de casos acumulados da COVID-19 com taxas acima e abaixo da média estadual (Tabela 4).

A pobreza foi mensurada pela parcela da população que possui uma renda domiciliar per capita mensal de até $\mathrm{R} \$ 140,00$, a valores de agosto de 2010 (6). É um indicador socioeconômico relacionado ao acesso do cidadão à recursos financeiros que são destinados aos gastos com alimentação, educação, despesas do domicílio (água, energia elétrica etc.) e outros custos relacionados a manutenção do bem-estar da família.

O distanciamento social foi uma estratégia adotada pelos governos municipais e estaduais como alternativa de conter o avanço do contágio do vírus da COVID-19 na população e consistiu basicamente na restrição temporária da realização de algumas atividades públicas e a ocorrência de aglomerações, como é o caso de escolas, comércio e serviços públicos não essenciais. Dessa forma, as pessoas foram estimuladas a ficarem em suas residências (16).

A partir da adoção do distanciamento social, observou-se que uma parte da população foi capaz de manter o vínculo empregatício e conseguiram conduzir as atividades profissionais de forma remota. Por sua vez, uma parte considerável da população não dispõe das condições mínimas para exercitar o distanciamento social (por fatores relacionados à restrição de renda e ausência de contrato formal de trabalho, por exemplo) e se enquadram os desempregados, trabalhadores informais e autônomos (16).

Um dos principais aspectos relacionados às medidas de distanciamento social nos municípios brasileiros, são as restrições impostas na circulação dos desempregados em busca de trabalho e aos trabalhadores informais e autônomos na comercialização de produtos e oferta de serviços na localidade ${ }^{16}$. A população mais pobre está empregada principalmente nos setores econômicos essenciais que não pararam durante o período de isolamento social e, por isso, em função da necessidade de locomoção para o trabalho, estão mais expostas aos riscos de contágio do vírus da COVID-19 $(17,18)$.

$\mathrm{O}$ principal impacto do isolamento social nos municípios sul-rio-grandenses e brasileiros para as famílias mais pobres é em relação à renda, o que torna relevante a adoção de políticas públicas de transferência de renda direta e medidas de proteção 
social (por exemplo, doação de alimentos) para os segmentos sociais mais vulneráveis durante o período de pandemia $(17,19)$. Além disso, a perda de renda das famílias mais pobres durante o período de isolamento social, pode trazer problemas relacionados à deficiência alimentar e nutricional dos membros familiares em função da restrição de acesso à alimentos essenciais a sobrevivência humana (20-22).

Outro indicador socioeconômico que contribuiu para a diferenciação dos grupos de municípios do Rio Grande do Sul com taxa de incidência de casos acumulados da COVID-19 (acima e abaixo da média estadual) foi a parte da população que não possui ensino fundamental completo e com ocupação na informalidade (Tabela 4). Os trabalhadores informais possuem uma probabilidade menor do que os trabalhadores formais de receber recursos financeiros advindos de políticas de proteção social (seguro-desemprego) e de encargos trabalhistas no caso de demissão. Além disso, os trabalhadores informais vivem em condições instáveis (incerteza quanto a possibilidade de obtenção de renda em determinado período, por exemplo) e raramente possuem reservas de emergência para suportar o período de crise provocada pela pandemia da COVID-19 ao cumprir as recomendações de distanciamento social (22).

Pela necessidade de obtenção de renda, os trabalhadores informais acabam não cumprindo plenamente as medidas de isolamento social e se expõem ao risco de contágio em busca de obtenção de recursos financeiros para o sustento da família (22). Numa avaliação da PNAD Contínua COVID-19, no mês de maio de 2020, o rendimento médio dos trabalhadores informais no Brasil foi de $\mathrm{R} \$ 1.092,12$, correspondendo a $60 \%$ do montante do que é recebido habitualmente no mesmo período (de aproximadamente $\mathrm{R} \$ 1.820,81$ ). O segmento econômico mais afetado pela pandemia no Brasil foi o de serviços com alto grau de informalidade, que é a de cabelereiros, trabalhadores de tratamento de beleza e serviços pessoais que receberam efetivamente apenas $43 \%$ $(\mathrm{R} \$ 656,00)$ da renda habitual $(\mathrm{R} \$ 1.542,62)(23)$.

No Rio Grande do Sul, o rendimento médio efetivo dos trabalhadores em maio de 2020 foi de $\mathrm{R} \$ 2.052,94$, que correspondeu a $82 \%$ da renda habitual ( $\mathrm{R} \$ 2.493,96)$. O auxílio emergencial, aprovado na Lei número 13.982 de 20 de abril de 2020, se tornou uma importante política pública de transferência de renda para a população mais vulnerável durante do período da pandemia do COVID-19. Durante o mês de maio de 2020, a única renda obtida em 3,5 milhões de domicílios brasileiros foi apenas do auxílio emergencial (23).

Sendo assim, ressalta-se que os impactos da pandemia do COVID-19 sobre os rendimentos efetivos do trabalho foram muito mais acentuados nos domicílios da população mais pobre e, os rendimentos de outras fontes, como a do auxílio emergencial, se tornou mais relevante para esse segmento social durante o período de crise sanitária e econômica no Brasil (23).

A variável INF possui como aspecto complementar o baixo nível de escolaridade da população com idade superior a 18 anos e essa realidade também contribuiu a diferenciação dos grupos de municípios sul-rio-grandenses com taxas de incidência de casos acumulados da COVID-19 acima e abaixo da média estadual (Tabela 4). Uma parte considerável da população pobre do Rio Grande do Sul e do Brasil possuem um baixo nível de escolaridade, e esse fator contribuirá para que esse grupo social tenha dificuldades em interpretar adequadamente as informações relacionadas ao vírus da COVID-19, como por exemplo, as formas de transmissão e as medidas preventivas para se evitar o contágio (24). Ressalta-se a importância da implementação de políticas educacionais em saúde para as pessoas mais vulneráveis socioeconomicamente como forma de evitar a expansão dos casos de COVID-19 na sociedade (25).

Em síntese, durante o período de pandemia da COVID-19 no Rio Grande do Sul, 
destacam-se as seguintes estratégias de intervenção governamental direcionadas as populações mais pobres, sendo: (a) comunicação, compartilhamento e monitoramento de informações sobre as medidas de isolamento social e de prevenção ao vírus; (b) garantia de segurança alimentar e nutricional; e, (c) provisão de renda emergencial, bem como o incentivo e potencialização de ações de geração de renda a partir dos negócios locais (26).

Em relação a garantia da segurança alimentar e nutricional da população vulnerável socioeconomicamente nos municípios sul-rio-grandenses, destacam-se as políticas públicas do Programa Nacional de Alimentação Escolar (PNAE) e o Programa de Aquisição de Alimentos (PAA). Em suma, recomendam-se a adoção de estratégias como a distribuição de kits ou refeições para as famílias dos alunos de escolas públicas durante o período de suspenção das aulas, ampliação dos recursos públicos destinados às políticas do PNAE e PAA após o período da pandemia para os municípios que apresentam um baixo Índice de Desenvolvimento Humano (IDH) (27-29).

Vale a pena destacar que o acesso aos serviços de abastecimento de água e coleta de esgoto, bem como a densidade por dormitório no domicílio superior a 2 não foram capazes de diferenciar os municípios gaúchos com taxas de casos acumulados da COVID-19 acima e abaixo da média estadual em virtude do Rio Grande do Sul apresentar indicadores socioeconômicos (saneamento básico, educação, saúde, etc) superiores à outras unidades federativas brasileiras $(9,10)$, como é o caso das regiões Norte e Nordeste.

Com isso, a população sul-rio-grandense, em geral, vive em melhor condição de características do domicílio e de acesso à serviços públicos de saneamento básico, saúde, educação e dentre outros em relação à de outras localidades brasileiras. Além disso, ressalta-se que uma das limitações da atual pesquisa é a possibilidade de subnotificação da taxa de casos acumulados da COVID-19 no estado do Rio Grande do Sul. Já que a rápida expansão de contágio pelo vírus (SARS-Cov2) no país e o baixo número de testes realizados podem dificultar a estimativa real da quantidade de casos confirmados (30).

\section{Considerações finais}

Os fatores socioeconômicos associados à expansão de casos de COVID-19 nos municípios do Rio Grande do Sul estão relacionados a renda, que são a taxa de pobreza e a parcela da população com idade superior a 18 anos que possui baixo nível de escolaridade e com ocupação na informalidade. Sendo assim, essa parte da população sul-rio-grandense está sujeita ao risco de contágio pelo vírus em virtude das situações de busca de oportunidade de emprego e de renda durante o período de pandemia, ao uso inadequado de equipamentos de proteção individual ou a falta de recursos para adquirilos e nas aglomerações residenciais devido à falta de informação ou a má interpretação das recomendações da OMS.

Com isso, atribui-se a importância de políticas públicas de transferência de renda direta para esse segmento social, como é o caso do auxílio emergencial, que consistiu na única fonte de renda de uma parte dos domicílios brasileiros durante o período de isolamento social, ações para a garantia da segurança alimentar e nutricional da população mais vulnerável socioeconomicamente, que consistem no PNAE e PAA, e políticas educacionais quanto à comunicação e compartilhamento de informações sobre as medidas de isolamento social e de prevenção ao vírus da COVID-19.

Dessa forma, recomenda-se a realização desse mesmo tipo de avaliação para outras unidades federativas e para o Brasil para verificar se outros aspectos socioeconômicos 
(acesso aos serviços de saneamento básico e aglomeração nos domicílios mensurada através da razão de dependência por dormitório, por exemplo), além da renda (como foi no Rio Grande do Sul), estão relacionados à expansão dos casos acumulados da COVID-19 ao longo do território nacional.

\section{Referências}

1. Garcia LP, Duarte E. Intervenções não farmacológicas para o enfrentamento à epidemia da COVID19 no Brasil. Epidemiologia e Serviços de Saúde 2020; 29(2): 1-4.

2. World Health Organization (WHO). Coronavirus disease (COVID-19) Pandemic. Geneva: WHO. 2020. [acessado 2020 Ago 06]. Disponível em: https://www.who.int/emergencies/diseases/novelcoronavirus-2019

3. Macedo YM, Ornellas JL, Bomfim HF. COVID-19 no Brasil: o que se espera para população subalternizada? Revista Encantar - Educação, Cultura e Sociedade 2020; 2:01-10.

4. Ministério da Saúde (MS). Coronavírus/Brasil. Brasília: MS. 2020. [acessado 2020 Ago 06]. Disponível em: https://covid.saude.gov.br/

5. Coelho FC, Lana RM, Cruz OG, Villela D, Bastos LS, Piontti AP, Davis JT, Vespignani A, Codeço CT, Gomes MFC. Assessing the potential impacto of COVID-19 in Brazil: mobility, morbidity and the burden on the health care system. medRxiv 2020; (preprint submitted to Elsevier). Disponível em: https://www.medrxiv.org/content/10.1101/2020.03.19.20039131v2

6. Instituto Brasileiro de Geografia e Estatística (IBGE). Censo Demográfico. Rio de Janeiro: IBGE. 2010. [acessado 2020 Ago 07]. Disponível em: https://www.ibge.gov.br/estatisticas/sociais/populacao/25089-censo-1991-6.html?=\&t=o-que-e

7. Werneck GL, Carvalho MS. A pandemia de COVID-19 no Brasil: crônica de uma crise sanitária anunciada. Cadernos de Saúde Pública 2020; 36(5): 1-4.

8. Secretária de Saúde do Estado do Rio Grande do Sul (SESRS). Painel coronavírus RS. Porto Alegre: SESRS. 2020. [acessado 2020 Ago 06]. Disponível em: https://ti.saude.rs.gov.br/covid19/

9. Tavares JM, Pôrto Junior SS. Análise das desigualdades inter e intraestaduais na região Sul do Brasil por meio da análise de componentes principais. Perspectiva Econômica 2011; 7(1): 1-14.

10. Lazaretti LR, Teixeira FO, Oliveira SV. Desigualdades socioeconômicas e demográficas nos municípios do Rio Grande do Sul/Brasil: um índice de vulnerabilidade à pobreza. Revista Brasileira de Gestão Urbana 2019; 11: 1-15.

11. Mário PC. Análise Discriminante. In: Corrar LJ, Paulo E, Dias Filho JM, coordenadores. Análise Multivariada para os cursos de Administração, Ciências Contábeis e Economia. São Paulo: Editora Atlas; 2007. p. 232-279.

12. Programa das Nações Unidas para o Desenvolvimento (PNUD). Atlas do Desenvolvimento Humano no Brasil. Nova Iorque: PNUD. 2020. [acessado 2020 Ago 03]. Disponível em: http://atlasbrasil.org.br/2013/pt/consulta/

13. Fundação Getúlio Vargas (FGV). Indicador Social de Desenvolvimento dos Municípios. São Paulo: FGV. 2020. [acessado 2020 Ago 07]. Disponível em: https://cmicro.fgv.br/data-sets

14. Secretária de Planejamento, Orçamento e Gestão do estado do Rio Grande do Sul (SEPLAG/RS). DEEdados. Porto Alegre: SEPLAG. 2020. [acessado 2020 Ago 07]. Disponível em: http://deedados.planejamento.rs.gov.br/feedados/\#!home/datacriacao

15. Hair JF, Black WC, Babin BJ, Anderson RE, Tatham RL. Análise multivariada de dados. São Paulo: Editora Bookman; 2009.

16. Pires RRC. Os efeitos sobre grupos sociais e territórios vulnerabilizados das medidas de enfrentamento à crise sanitária da covid-19 propostas para o aperfeiçoamento da ação pública: Nota Técnica Brasília: IPEA; 2020. [acessado 2020 Ago 08]. Disponível em: http://repositorio.ipea.gov.br/bitstream/11058/9839/1/NT_33_Diest_Os\%20Efeitos\%20Sobre\%20Gr upos\%20Sociais\%20e\%20Territ\%c3\%b3rios\%20Vulnerabilizados.pdf

17. Bezerra ACV, Silva CEM, Soares FRG, Silva JAM. Fatores associados ao comportamento da população durante o isolamento social na pandemia de COVID-19. Ciência e Saúde Coletiva 2020; 25(Supl. 1): 2411-2421. 
18. Bonaccorsi G, Pierri F, Cinelli M, Flori A, Galeazzi A, Porcelli F, Schmidt AL, Valensise CM, Scala A, Quattrociocchi W, Pammolli F. Economic and social consequences of human mobility restrictions under COVID-19. PNAS 2020; 117(27): 15530-15535.

19. Krishnakumar B, Rana S. COVID-19 in INDIA: Strategies to combat from combination threat of life and livelihood. Journal of Microbiology, Immunology and Infection 2020; 53(3): 389-391.

20. Shammi M, Bodrud-Doza M, Islam ARMT, Rahman MM. COVID-19 pandemic, socioeconomic crisis and human stress in resource-limited settings: a case from Bangladesh. Heliyon 2020; 6(5):112.

21. Barker M, Russell J. Feeding the food insecure in Britain: learning from the 2020 COVID-19 crisis. Food Security 2020.

22. Sakamoto M, Begum S, Ahmed T. Vulnerabilities to COVID-19 in Bangladesh and a reconsideration of sustainable development goals. Sustainability 2020; 12(13): 5296.

23. Carvalho SS. Os efeitos da pandemia sobre os rendimentos do trabalho e o impacto do auxílio emergencial: o que dizem os microdados da PNAD covid-19: Carta de Conjuntura Brasília: IPEA; 2020. [acessado 2020 Ago 10]. Disponível em: https://www.ipea.gov.br/portal/images/stories/PDFs/conjuntura/200702_cc_48_mercado_de_trabalho .pdf

24. Ribeiro F, Leist A. Who is going to pay the price of COVID-19? Reflections about na unequal Brazil. International Journal for Equity in Health 2020; 19:91.

25. Lau LL, Hung N, Go DJ, Ferma J, Choi M, Dodd W, Wei X. Knowledge, atitudes and practices of COVID-19 among income-poor households in the Philippines: a cross-sectional study. Journal of Global Health 2020; 10(1): 011007.

26. Perissé A, Leandro BB, Batistella CC, Barcellos C, Santos JL, Angelo JR, Cunha M, Dominguez M, Carrijo RSGG, Gondim R. Covid-19 e vulnerabilidades: considerações sobre proteção social nas favelas: Nota Técnica Rio de Janeiro: Fiocruz; 2020. [acessado 2020 Ago 10]. Disponível em: https://www.arca.fiocruz.br/bitstream/icict/41456/2/Covid-19Vulnerabilidades.pdf

27. Amorim ALB, Ribeiro Junior JRS, Bandoni DH. Programa Nacional de Alimentação Escolar: estratégias para enfrentar a insegurança alimentar durante a após a COVID-19. Revista de Administração Pública 2020; 54(4): 1134-1145.

28. Sambuichi RHR, Almeida AFCS, Perin G, Spínola PAC, Pella AFC. O Programa de Aquisição de Alimentos (PAA) como estratégia de enfrentamento aos desafios da COVID-19. Revista de Administração Pública 2020; 54(4): 1079-1096.

29. Araújo FR, Calazans DLMS. Gestão de ações de segurança alimentar frente à pandemia pela COVID-19. Revista de Administração Pública 2020; 54(4): 1123-1133.

30. Prado MF, Antunes BBP, Bastos LSL, Peres IT, Silva AAB, Dantas LF, Baião FA, Maçaira P, Hamacher S, Bozza FA. Analysis of COVID-19 under-reporting in Brazil. Revista Brasileira de Terapia Intensiva; 32(2): 224-228. 